\title{
Practice-changing Clinical Studies in Prostate Cancer: an Update for the Medical Oncologist
}

\author{
Laura Cristina Zaharie ${ }^{1}$ \\ 1 Oncology Institute "Prof. Dr. Ion. Chiricuță”, Cluj-Napoca, Romania
}

Corresponding author: Laura Cristina Zaharie; e-mail: Ic.zaharie@yahoo.com

\begin{abstract}
With new updates every year on cancer diagnosis, treatment, and follow-up, the face of oncology is changing rapidly. With the new guidelines issued this year and, also, with the recently published phase III trials' results, we aim to summarize key changes and updates for prostate cancer patients. We searched PubMed and international societies' databases for recent articles and chose those with relevant information for everyday clinical practice. Our review includes assessments for specific diagnostic methods and the most recent treatment options available for prostate cancer.

Keywords: review, prostate cancer, guidelines, clinical practice
\end{abstract}

\section{Introduction}

Prostate cancer $(P C)$ is the fourth most common cancer and the second most frequent cancer in men, and is one of the leading causes of cancer-related deaths worldwide and the fourth cause of cancer-related death in the USA in 2020 (1). Recently, the European Society of Medical Oncology (ESMO) and the European Association of Urology (EAU), as well as the National Comprehensive Cancer Network (NCCN) updated their guidelines on screening, diagnosis, treatment and follow-up of prostate cancer patients.

\section{Methods}

Besides the European and American guidelines, we conducted a search in the PubMed database based on the keywords "prostate cancer", "metastatic", "hormonal therapy", "castration", and "immunotherapy". We selected papers published in the last two years and included phase III trials and studies with a significant number of participants, based on the relevance of the information and the applicability in real-life situations. 


\section{Discussion}

\subsection{Workup}

It is commonly stated that PSA screening of men decreases cancer mortality but also leads to unacceptable overdiagnosis and significant overtreatment. Thus, current recommendations suggest using this method of screening in men over 50 years or over 45 years in case of positive family history $(2,3)$.

The main change regarding workup is the recommendation (class IB) of performing multiparametric magnetic resonance imaging (mpMRI) before prostate biopsy. A positive mpMRI is defined by a PIRADS score of at least 3 . However, there is growing concern that the increased use of mpMRI would lead to more unnecessary biopsies and overdiagnosis of PC. Risk calculator models such as the recently validated Rotterdam Prostate Cancer Risk Calculator and the globally recognized STAR-CAP, are increasingly used to better predict the outcomes of and adapt treatment (4). When indicated, targeted transperineal MRI fusion biopsies proved to have an increased detection rate of clinically significant $P C$ and fewer adverse effects compared to transrectal biopsies.

Tumor extension assessed by MRI, Gleason score and initial PSA values are used to classify prostate cancers as low risk, intermediate-risk or high-risk disease (5). In the most recent guidelines published by ESMO, the low-risk category refers to men with a T1/2 stage, a Gleason score $\leq 6$ and PSA less than 10. In this case, no further imaging method is required. For the intermediate or high-risk categories, further evaluation of nodal or distant metastatic disease using at least a bone scan, a whole-body MRI, a CT/PET

CT or, if available, prostate-specific membrane antigen (PSMA)-PET-CT is recommended (6). Currently, the emergent method of liquid biopsies is of great interest in the process of cancer diagnosis, therapy, prognosis and follow-up. It is dramatically changing modern oncology by analyzing cir- culating tumor cells and circulating cell-free DNA (cfDNA), with the latter containing circulating tumor DNA (ctDNA) in cancer patients. cfDNA refers to extracellular DNA which originates from tumor-associated necrosis or physiological apoptosis. ctDNA may have similar genetic alterations with both the primary tumor and its distal metastases, and represents an important source of information. A study by Barata et al. has given a novel perspective on the direct clinical implications of integrating liquid biopsies into general practice. (7).

Recent studies have proved that cfDNA is a promising biomarker for screening and early detection of PC (8). Also, a study conducted by Lau et al. concluded that detecting ctDNA in patients with clinically localized PC can be used as a prognostic marker as it is associated with rapid disease progression (9). However, Hennigan et al. have demonstrated that the blood of patients with localized disease has a lower quantity of ctDNA, making it more difficult to assess (10). As shown by an editorial published by EMSO in 2021, a multitude of unknown variables that need further research remain (11).

\subsection{Hormonal and Chemotherapy}

Surgery and/or radiotherapy (RT) are the main therapeutic options for localized PC. For patients with low-risk disease, active surveillance is a Class IA recommendation. The ProtecT trial compared active therapy versus monitoring and there was no statistically significant difference after a 10year follow-up (12). In patients with high-risk disease, the best approach based on two trials would be RT in association with androgen deprivation therapy (ADT) or radical prostatectomy (RP) (13). In case of bio chemical relapse of the disease after RP, the current recommendations are salvage radiotherapy with at least $66 \mathrm{~Gy}$ alongside ADT, which is known for its radio-sensitizing effect (14). A phase III trial, GETUG-AFU 17, has shown that adjuvant radiotherapy has no benefit for event-free survival in patients compared to early salvage radiotherapy 
(SRT) (15). Early SRT should be given immediately after the first sign of PSA rise (16).

A high number of studies have been conducted in order to investigate the useful ness of neoadjuvant or adjuvant hormone treatment. Several trials have shown the benefit of adding neoadjuvant ADT to highrisk $\mathrm{PC}$ and, also, to unfavorable intermediate-risk localized PC. Docetaxel-based chemotherapy in high-risk localized disease has been proven in a meta analysis based on CHAARTED, GETUG-15 and STAMPEDE trials to improve the relapse-free survival rate by $8 \%(17,18)$. Regarding metastatic hormone-naïve PC, two phase III trials, CHAARTED and STAMPEDE, established the benefit of docetaxel in combination with ADT for these patients, as it significantly improved overall survival (OS) from 47.2 months to 57.6 months $(19,20)$.

Another study conducted by Roy et al. evaluated the impact of concomitant medications on biochemical outcome in localized PC patients. Metformin intake doubled the risk of biochemical relapse, while sulfonamide-based concomitant alpha blockers nearly tripled risk (21).

For over half a century, androgen deprivation therapy was the first line of treatment for metastatic PC, after LHRH agonists replaced surgical castration as the gold standard. Recently, the androgen axis was effectively targeted with new therapies such as androgen receptor-targeted agents (apalutamide, enzalutamide) and a CYP17 inhibitor (abiraterone) (22). Patients with a low volume disease should be offered the combination of ADT with prostate RT as suggested by CHAARTED trial's criteria (20).

LATITUDE and STAMPEDE have demonstrated that the addition of abiraterone to ADT also improves overall survival by $7 \%$, while another phase III trial, TITAN, has demonstrated that apalutamide has a significant improvement in 2-year-OS. Enzalutamide has been shown by two trials (ENZAMET and ARCHES) to have an $8 \%$ improvement in 3-year-OS data (23). Taking into consideration that docetaxel and abiraterone can produce adverse effects with greater impact on quality of life, they should be offered to compliant patients with fewer comorbidities and good performance status.

Castration-resistant prostate cancer (CRPC) refers to disease progression (biochemical or radiological) under ADT and having serum testosterone at castrate levels, meaning $<50 \mathrm{ng} / \mathrm{dl}$. Three trials (SPARTAN, PROSPER and ARAMIS), tested a modern antiandrogen combined with ADT, and have shown that apalutamide, enzalutamide or darolutamide bring improvements to overall survival in both metastatic-free and non-metastatic castration resistant prostate cancer (M0- CRPC) patients (Table 1) (24).

Unfortunately, there are also many patients that are diagnosed with metastatic CRPC de novo. Recent studies have emphasized the importance of genetic counseling and testing for germline mutations, at least for patients with strong family history, with hereditary breast and ovarian cancer syndrome and Lynch syndrome and with high risk PC or intraductal histology (25).

For these patients, first-line treatment options include abiraterone, enzalutamide and docetaxel-based chemotherapy. The first two can be starting options for asymptomatic or mildly symptomatic patients with chemotherapy-naïve metastatic castration resistant prostate cancer (mCRPC), while docetaxel should be the first option for those with rapidly progressing disease or predominantly visceral metastases (2). In case of disease progression under chemotherapy, the treatment options are the second AR targeted drugs or cabazitaxel, which appears to be superior and, moreover, has recently been approved in Romania (24). In the CARD study, the median PFS for cabazitaxel was 8 months compared to 3.7 months for abiraterone or enzalutamide and the median OS was 13.6 months vs 11.0 months (26). 
Table 1. Combined androgen blockade for MO-CRPC

\begin{tabular}{|l|l|l|l|}
\hline & $\begin{array}{l}\text { SPARTAN } \\
\text { Apalutamide vs } \\
\text { placebo }\end{array}$ & $\begin{array}{l}\text { PROSPER } \\
\text { Enzalutamide vs } \\
\text { placebo }\end{array}$ & $\begin{array}{l}\text { ARAMIS } \\
\text { Darolutamide vs } \\
\text { placebo }\end{array}$ \\
\hline $\mathbf{n}$ & 806 vs 401 & 933 vs 468 & 955 vs 554 \\
\hline $\begin{array}{l}\text { Metastasis free survival } \\
(\mathbf{m o})\end{array}$ & 40.5 vs $16.2(p<0.001)$ & 36.6 vs $14.7(p<0.001)$ & 40.4 vs $18.4(p<0.001)$ \\
\hline Overall survival (mo) & 73.9 vs $59.9(p<0.001)$ & 67.0 vs $56.3(p<0.001)$ & NR vs NR \\
\hline
\end{tabular}

$\mathrm{n}$ - number of patients; mo - months; NR - not reached

Regarding a second-line treatment for $\mathrm{mCRPC}$, it is important to mention that there is evidence of cross resistance between enzalutamide and abiraterone and current guidelines do not recommend the use of a second AR inhibitor (27).

An important trial, ALSYMPCA, has emphasized that for a progressive bone-pre dominant symptomatic $\mathrm{mCRPC}$, radium223, a bone-targeted alpha-emitter, significantly increased OS. However, its use is restricted to patients who are ineligible for firstline therapies or still have progressive disease after two lines of systemic treatment (28).

This year, Mao et al. published a study regarding the synergistic antitumor effect of the ZD55-IL-24 oncolytic adenovirus combined with radiotherapy in $\mathrm{PC}$ in vitro and in vivo trials. They found that this combination can induce apoptosis in tumoral cells by increasing their radiosensitivity and inhibit angiogenesis (29).

\subsection{Immunotherapy}

In recent years, immune checkpoint inhibitor therapy has been correlated with a significant improvement in the survival of patients with solid tumors. Unfortunately, data on $\mathrm{mCRPC}$ remains unclear and needs further investigation as prostate cancer appears to be an immunologically "cold" tumor (30). Recent results indicate that $\mathrm{mCRPC}$ has a low response to immunotherapy. This may be related to several factors: low tumor mutational bur den, loss of tumor suppressor gene, low prevalence of genetic defects and silencing of major histocompatibility complex-1 (31).

Although the development of immune checkpoint monoclonal antibodies represents a breakthrough in cancer therapy, the detection of specific biomarkers that predict treatment response remains a dynamic area of research in oncology. The most commonly used biomarker nowadays is the quantitative expression of PD-L1, which has been reported to have a significant expression for both mCRPC and primary PC (30). Vicier et al. published a study that assessed the PD-L1 expression in men with PC after undergoing prostatectomy and concluded that a lower expression is associated with a lower risk of biochemical relapse and presence of metastasis (32).

Recent data have shown that there are special populations of PC patients who can benefit from immunotherapy and these are represented by the patients with $\mathrm{MSI}-\mathrm{H}$ tumors (33).

According to Willi et al., enzyme deficiency can lead to microsatellite instability (MSI) in cancer, and is associated with high levels of mutation neoantigens, but only 2$3 \%$ of PC cases have been shown to have high microsatellite instability (MSI-H) tumors (34). Barataet al. reported the first case series of clinical activity of pembroli zumab for MSI-H mCRPC. In this study, durable and profound responses were observed in nearly half of the MSI-H tumors (7).

Another study published by Graff et al. has shown evidence that ipilimumab, a fully 
human monoclonal antibody promoting antitumor activity, can increase antitumoral activity and progression-free survival in patients with $\mathrm{MCRPC}$, although without an improvement in overall survival (35).

\subsection{Other genetic-alteration driven therapy}

An increased number of DNA errors leading to high tumor neoantigen expression has been observed in patients with mutations in the DNA damage repair genes (BRCA1, BRCA2, CDK12, ATM) $(36,37)$.

For patients with genetic mutations, a new class of drugs has been introduced, known as poly-adenosine diphosphate-ribose polymerase (PARP) inhibitors $(31,38)$.

There are several ongoing trials for PARP inhibitors alone or in various combi nations for mCRPC. Currently, two drugs, olaparib and rucaparib are approved as a new line of treatment for patients who have a progressive disease after first and second generation of androgen receptor axis-targeting agents, taxane-based chemotherapy, radium-223 and immunotherapy with Sipuleucel-T or pembrolizumab (38). The recent PROfound trial, a prospective randomized phase III trial, screened 4425 patients with mCRPC and found that 778 had qualifying alterations (39).

\subsection{Future directions}

Considering the recent technological advancements in artificial intelligence, technology is becoming more and more involved in solving medical dilemmas.

There is a great interest shown in developing and validating a deep learning algorithm for Gleason grading of PC. In a recent study published in JAMA Oncology, Nagpal et al. presented a deep learning system (DLS) for reading digital prostate biopsy specimen sections. The results show a similar rate of identifying tumor specimens between DLS and pathologists, as well as significantly better agreement rates on Gleason pattern vs. pathologists. Considering the high interobserver variability in Gleason grading, DLS could function as a decision tool, with significant therapeutic implications streamlining the diagnostic flow (40).

Recently, a machine learning based analysis has been developed in order to predict metastatic disease or high-risk pathological tumor features in primary $\mathrm{PC}$ with the use of ${ }^{18}[\mathrm{~F}] \mathrm{DCFPyL}$ PET metrics. Lymph node involvement for these patients is an important diagnostic finding. Currently their involvement in cancer is investigated with an extended pelvic lymph node dissection. Prostate-specific membrane antigen is a type-Il transmembrane protein highly over expressed on PC cells. Therefore, quantitative measures of its expression are widely used as a biomarker for risk stratification. The machine learning algorithm developed by Cysouw et al. was able to predict disease risk in primary $P C$ patients and they stated that PSMA PET radiomics may capture tumor aggressiveness by carrying genomic as well as histopathological information (41).

\section{Conclusion}

Prostate cancer treatment is rapidly evolving. The latest guidelines are intended to offer patients the best diagnostic and treatment options. Our brief review offers clinicians several tips on the 2020-2021 updates for the management of prostate cancer.

\footnotetext{
Abbrevations:

PC - Prostate cancer

ESMO - European Society of Medical Oncology

EAU - European Association of Urology

NCCN - National Cancer Comprehensive Network
} 
mpMRI - multiparametric magnetic resonance imaging

cfDNA - circulating cell-free DNA

ctDNA - circulating tumor DNA

$\mathrm{RT}$ - radiotherapy

ADT - androgen deprivation therapy

$\mathrm{RP}$ - radical prostatectomy

SRT - salvage radiotherapy

OS - overall survival

CRPC - castration-resistant prostate cancer

mCRPC - metastatic castration resistant prostate cancer

$\mathrm{MSI}$ - microsatellite instability

MSI-H - high microsatellite instability

PARP - poly-adenosine diphosphate-ribose polymerase

DLS - deep learning system

CT- computed tomography

PET - CT-positron emission tomography-computed tomography

$\mathrm{MRI}$ - magnetic resonance imaging

PFS - progression free survival

AR - androgen receptor

PD-L1 - program-death ligand 1

Statements: None.

Previous publication: I declare that this paper was not published nor was submitted to be reviewed for publication in another journal.

Conflict of interest: I declare having no competing interests associated with this publication.

Funding Sources: This research did not receive any specific grant from funding agencies in the public, commercial, or not-for-profit sector.

\section{References}

1. CANCER FACT SHEETS: PROSTATE CANCER [Internet]. [cited 2020 Dec 10]. Available from: http://gco.iarc.fr/today

2. Parker C, Castro E, Fizazi K, Heidenreich A, Ost P, Procopio G, et al. Prostate cancer: ESMO Clinical Practice Guidelines for diagnosis, treatment and follow-up†. Ann Oncol [Internet]. 2020;31(9):1119-34. Available from: https://doi.org/10.1016/j.annonc.2020.06.011

3. Mottet N, Bastian P, Bellmunt J, van den Bergh R, Bolla M, van Casteren N, et al. EAU - EANM - ESTRO ESUR - SIOG: Guidelines on Prostate Cancer. Eur Assoc Urol. 2020;(April):1-182.

4. Tully KH, Bahlburg H, Berg S, Hanske J, von Landenberg N, Noldus J, et al. Changing the Prostate Cancer Detection Paradigm: Clinical Application of European Association of Urology Guideline-recommended Magnetic Resonance Imaging-based Risk Stratification in Men with Suspected Prostate Cancer. Eur Urol Focus. 2020;1-8.

5. Joniau S, Briganti A, Gontero P, Gandaglia G, Tosco L, Fieuws S, et al. Stratification of high-risk prostate cancer into prognostic categories: A european multi-institutional study. Eur Urol [Internet]. 2015;67(1):157-64. Available from: http://dx.doi.org/10.1016/j.eururo.2014.01.020

6. Carlsson S, Castle EP, Catalona WJ, Robert Lurie $\omega \mathrm{H}$, Dahl DM, Davis JW, et al. NCCN Guidelines Version 2.2020 Prostate Cancer Early Detection. 2020.

7. Barata P, Agarwal N, Nussenzveig R, Gerendash B, Jaeger E, Hatton W, et al. Clinical activity of pembrolizumab in metastatic prostate cancer with microsatellite instability high $(\mathrm{MSI}-\mathrm{H})$ detected by circulating tumor DNA. J Immunother Cancer. 2020;8(2):8-12. 
8. Ponti G, Maccaferri M, Percesepe A, Tomasi A, Ozben T. Liquid biopsy with cell free DNA: new horizons for prostate cancer. Crit Rev Clin Lab Sci [Internet]. 2020;0(0):1-17. Available from: https://doi.org/10.1080/10408363.2020.1803789

9. Lau E, McCoy P, Reeves F, Chow K, Clarkson M, Kwan EM, et al. Detection of ctDNA in plasma of patients with clinically localised prostate cancer is associated with rapid disease progression. Genome Med. 2020;12(1):1-11.

10. Hennigan ST, Trostel SY, Terrigino NT, Voznesensky OS, Schaefer RJ, Whitlock NC, et al. Low Abundance of Circulating Tumor DNA in Localized Prostate Cancer. JCO Precis Oncol. 2019;(3):1-13.

11. Jayaram A, Wingate A, Wetterskog D, Wheeler G, Sternberg CN, Jones R, et al. Plasma tumor gene conversions after one cycle abiraterone acetate for metastatic castration-resistant prostate cancer: a biomarker analysis of a multicenter international trial. Ann Oncol [Internet]. 2021;32(6):726-35. Available from: https://doi.org/10.1016/j.annonc.2021.03.196

12. Hamdy FC, Donovan JL, Lane JA, Mason M, Metcalfe C, Holding P, et al. 10-Year Outcomes after Monitoring, Surgery, or Radiotherapy for Localized Prostate Cancer. N Engl J Med. 2016;375(15):1415-24.

13. Widmark A, Klepp O, Solberg A, Damber JE, Angelsen A, Fransson P, et al. Endocrine treatment, with or without radiotherapy, in locally advanced prostate cancer (SPCG-7/SFUO-3): an open randomised phase III trial. Lancet [Internet]. 2009;373(9660):301-8. Available from: http://dx.doi.org/10.1016/S0140-6736(08)61815-2

14. Warde $\mathrm{P}$, Mason M, Ding K, Kirkbride P, Brundage M, Cowan R, et al. Combined androgen deprivation therapy and radiation therapy for locally advanced prostate cancer: A randomised, phase 3 trial. Lancet [Internet]. 2011;378(9809):2104-11. Available from: http://dx.doi.org/10.1016/S0140-6736(11)61095-7

15. Sargos P, Chabaud S, Latorzeff I, Magné N, Benyoucef A, Supiot S, et al. Adjuvant radiotherapy versus early salvage radiotherapy plus short-term androgen deprivation therapy in men with localised prostate cancer after radical prostatectomy (GETUG-AFU 17): a randomised, phase 3 trial. Lancet Oncol [Internet]. 2020;21(10):1341-52. Available from: http://dx.doi.org/10.1016/S1470-2045(20)30454-X

16. Sargos P, Chabaud S, Latorzeff I, Magné N, Benyoucef A, Supiot S, et al. Salvage Radiotherapy for Prostate Cancer. Clin Oncol [Internet]. 2020;21(10):156-62. Available from: https://doi.org/10.1016/j.clon.2020.01.003

17. Vale CL, Burdett S, Rydzewska LHM, Albiges L, Clarke NW, Fisher D, et al. Addition of docetaxel or bisphosphonates to standard of care in men with localised or metastatic, hormone-sensitive prostate cancer: A systematic review and meta-analyses of aggregate data. Lancet Oncol [Internet]. 2016;17(2):243-56. Available from: http://dx.doi.org/10.1016/S1470-2045(15)00489-1

18. von Amsberg G. Addition of docetaxel to hormonal therapy in low- and high-burden metastatic hormone sensitive prostate cancer: long-term survival results from the STAMPEDE trial. Urol . 2020;59(6):723-4.

19. Parker CC, James ND, Brawley CD, Clarke NW, Hoyle AP, Ali A, et al. Radiotherapy to the primary tumour for newly diagnosed, metastatic prostate cancer (STAMPEDE): a randomised controlled phase 3 trial. Lancet [Internet]. 2018;392(10162):2353-66. Available from: http://dx.doi.org/10.1016/S0140-6736(18)32486-3

20. Kyriakopoulos CE, Chen YH, Carducci MA, Liu G, Jarrard DF, Hahn NM, et al. Chemohormonal therapy in metastatic hormone-sensitive prostate cancer: long-term survival analysis of the randomized phase III E3805 chaarted trial. J Clin Oncol. 2018;36(11):1080-7.

21. Roy S, Malone S, Grimes S, Morgan SC. Impact of Concomitant Medications on Biochemical Outcome in Localised Prostate Cancer Treated with Radiotherapy and Androgen Deprivation Therapy. Clin Oncol [Internet]. 2020;(xxxx). Available from: https://doi.org/10.1016/j.clon.2020.09.005

22. Lam TBL, MacLennan S, Willemse PPM, Mason MD, Plass K, Shepherd R, et al. EAU-EANM-ESTRO-ESURSIOG Prostate Cancer Guideline Panel Consensus Statements for Deferred Treatment with Curative Intent for Localised Prostate Cancer from an International Collaborative Study (DETECTIVE Study). Eur Urol. 2019;76(6):790-813.

23. Sigurdson SS, Vera-Badillo FE, Moraes FY de. Discussion of Treatment Options for Metastatic Hormone Sensitive Prostate Cancer Patients. Front Oncol. 2020;10(October):11-4.

24. Cornford P, van den Bergh RCN, Briers E, den Broeck T Van, Cumberbatch MG, De Santis M, et al. EAU- 
EANM-ESTRO-ESUR-SIOG Guidelines on Prostate Cancer. Part II-2020 Update: Treatment of Relapsing and Metastatic Prostate Cancer. Eur Urol. 2020;1-20.

25. Shore ND, Drake CG, Lin DW, Ryan CJ, Stratton KL, Dunshee C, et al. Optimizing the management of castration-resistant prostate cancer patients: A practical guide for clinicians. Prostate. 2020;80(14):1159-76.

26. de Wit R, de Bono J, Sternberg CN, Fizazi K, Tombal B, Wülfing C, et al. Cabazitaxel versus Abiraterone or Enzalutamide in Metastatic Prostate Cancer. N Engl J Med [Internet]. 2019 Sep 30;381(26):2506-18. Available from: https://doi.org/10.1056/NEJMoa1911206

27. Moses M, Niu A, Lilly MB, Hahn AW, Nussenzveig R, Ledet E, et al. Circulating-tumor DNA as predictor of enzalutamide response post-abiraterone treatment in metastatic castration-resistant prostate cancer. Cancer Treat Res Commun [Internet]. 2020;24:100193. Available from: https://doi.org/10.1016/j.ctarc.2020.100193

28. Parker CC, Coleman RE, Sartor O, Vogelzang NJ, Bottomley D, Heinrich D, et al. Three-year Safety of Radium-223 Dichloride in Patients with Castration-resistant Prostate Cancer and Symptomatic Bone Metastases from Phase 3 Randomized Alpharadin in Symptomatic Prostate Cancer Trial. Eur Urol. 2018;73(3):427-35.

29. Mao LJ, Kan Y, Li BH, Ma S, Liu Y, Yang DL, et al. Combination Therapy of Prostate Cancer by Oncolytic Adenovirus Harboring Interleukin 24 and lonizing Radiation. Front Oncol. 2020;10(April):1-8.

30. Rizzo A, Mollica V, Cimadamore A, Santoni M, Scarpelli M, Giunchi F, et al. Is There a Role for Immunotherapy in Prostate Cancer? Cells. 2020;9(9):1-22.

31. Powers E, Karachaliou GS, Kao C, Harrison MR, Hoimes CJ, George DJ, et al. Novel therapies are changing treatment paradigms in metastatic prostate cancer. J Hematol Oncol [Internet]. 2020;13(1):1-13. Available from: https://doi.org/10.1186/s13045-020-00978-z

32. Vicier C, Ravi P, Kwak L, Werner L, Huang Y, Evan C, et al. Association between CD8 and PD-L1 expression and outcomes after radical prostatectomy for localized prostate cancer. Prostate [Internet]. 2021 Jan 1;81(1):50-7. Available from: https://doi.org/10.1002/pros.24079

33. Abida W, Cheng ML, Armenia J, Middha S, Autio KA, Vargas HA, et al. Analysis of the Prevalence of Microsatellite Instability in Prostate Cancer and Response to Immune Checkpoint Blockade. JAMA Oncol. 2019;5(4):471-8.

34. Willis J, Lefterova MI, Artyomenko A, Kasi PM, Nakamura Y, Mody K, et al. Validation of microsatellite instability detection using a comprehensive plasma-based genotyping panel. Clin Cancer Res. 2019;25(23):7035-45.

35. Graff JN, Stein MN, Surana R, Al Rabadi L, Liu E, Fong L, et al. Phase II Study of Ipilimumab in Men With Metastatic Prostate Cancer With an Incomplete Response to Androgen Deprivation Therapy. Front Oncol. 2020;10(August):1-9.

36. López-Campos F, Linares-Espinós E, Maldonado Pijoan X, Sancho Pardo G, Morgan TM, MartínezBallesteros C, et al. Genetic testing for the clinician in prostate cancer. Expert Rev Mol Diagn [Internet]. 2020;20(9):933-46. Available from: https://doi.org/10.1080/14737159.2020.1816170

37. Pritchard CC, Morrissey C, Kumar A, Zhang X, Smith C, Coleman I, et al. Complex MSH2 and MSH6 mutations in hypermutated microsatellite unstable advanced prostate cancer. Nat Commun. 2014;5:3-8.

38. Risdon EN, Chau CH, Price DK, Sartor O, Figg WD. PARP Inhibitors and Prostate Cancer: To Infinity and Beyond BRCA . Oncologist. 2020;1-15.

39. de Bono J, Mateo J, Fizazi K, Saad F, Shore N, Sandhu S, et al. Olaparib for Metastatic Castration-Resistant Prostate Cancer. N Engl J Med. 2020;382(22):2091-102.

40. Nagpal K, Foote D, Tan F, Liu Y, Chen PHC, Steiner DF, et al. Development and Validation of a Deep Learning Algorithm for Gleason Grading of Prostate Cancer from Biopsy Specimens. JAMA Oncol. 2020;6(9):1372-80.

41. Cysouw MCF, Jansen BHE, van de Brug T, Oprea-Lager DE, Pfaehler E, de Vries BM, et al. Machine learning-based analysis of [18F]DCFPyL PET radiomics for risk stratification in primary prostate cancer. Eur J Nucl Med Mol Imaging. 2020. 